\title{
Pancreatic Cystic Lymphangioma: Case Report and Literature Review
}

\author{
Sujata Ojha ${ }^{\mathrm{a}}$, Muhammad Darwish ${ }^{\mathrm{b}}$, Patrick McLaren ${ }^{\mathrm{b}}$, Annie Benzie $^{\mathrm{b}}$, Edward Cho ${ }^{\mathrm{b}, \mathrm{c}}$, \\ Houssam Osman ${ }^{\mathrm{b}}$, Dhiresh Rohan Jeyarajah ${ }^{\mathrm{b}, \mathrm{c}, \mathrm{d}}$
}

\begin{abstract}
Lymphangiomas are rare, benign tumors of the lymphatic system. They are formed through congenital malformation leading to lymphangiectasias. Thus, lymphangiomas are mostly seen in children and infants. Lymphangiomas are a benign lesion, therefore, if the patient is asymptomatic and the lesion is stable, complete surgical resection is not required and imaging surveillance is the preferred method of follow-up. This study reports a rare presentation of lymphangioma in the pancreas.
\end{abstract}

Keywords: Lymphangioma; Pancreatic lymphangioma; Pancreatic cyst; CT; Endosonography; Cystic

\section{Introduction}

Lymphangiomas are rare tumors of the lymphatic system that are regarded as a congenital malformation arising from proliferation of sequestered lymphatic tissue. They can also be acquired as a result of fibrosis, trauma, inflammation, or radiotherapy [1]. These benign tumors occur as a result of blockage of lymphatic flow leading to an abnormal dilation of lymph vessels, called lymphangiectasias [2]. Lymphangiomas are generally found in children with $75 \%$ of cases presenting in the head and neck region, followed by $15 \%$ of cases in the axillary region [3]. Pancreatic lymphangiomas are rare, accounting for less than $0.2 \%$ of all pancreatic cysts, and are more common in female patients $[4,5]$. They are generally asymptomatic and are incidentally found on imaging for non-pancreatic disease [5]. However, patients can present with abdominal pain and an

Manuscript submitted December 28, 2020, accepted January 30, 2021

Published online February 26, 2021

${ }^{a}$ TCU/UNTHSC School of Medicine, Fort Worth, TX, USA

bDepartment of Surgery, Methodist Richardson Medical Center, Richardson, TX, USA

'Department of Surgery, TCU/UNTHSC School of Medicine, Fort Worth, TX, USA

${ }^{\mathrm{d} C o r r e s p o n d i n g ~ A u t h o r: ~ D h i r e s h ~ R o h a n ~ J e y a r a j a h, ~ D e p a r t m e n t ~ o f ~ S u r g e r y, ~}$ Methodist Richardson Medical Center, 2805 East President George Bush Highway, Richardson, TX 75082, USA. Email: rohanjeyarajah@gmail.com

doi: https://doi.org/10.14740/jcs432 associated abdominal mass $[2,6]$. We present a case of pancreatic lymphangioma that was discovered incidentally.

\section{Case Report}

\section{Investigations}

The patient is a 65 -year-old female who presented with nausea and occasional abdominal pain. An abdominal computed tomography (CT) demonstrated an 8-cm cystic lesion involving the head of the pancreas with septation. Patient had no history of heavy alcohol use or previous attacks of pancreatitis. Laboratory findings were all within normal limits. Carbohydrate antigen 19-9 (CA 19-9) tumor marker was negative (4.27 U/mL).

\section{Diagnosis and treatment}

In order to obtain a definitive diagnosis, the patient was evaluated with an esophagogastroduodenoscopy (EGD)/endoscopic ultrasound (EUS) combined with a fine-needle aspiration (FNA) and computed tomography of chest abdomen pelvis (CT-CAP).

CT-CAP revealed an $8.1 \times 4.5 \times 5.6 \mathrm{~cm}$ cystic mass associated with the superior and anterior pancreatic body (Figs. $1,2)$. The superior anterior pancreatic body had a predominantly macrocytic mass lesion without evidence of adjacent organ invasion or any significant vascular encasement. The mass abutted the hepatic artery without evidence of invasion. The lesion was multiloculated with few thin septations and no wall thickening or mural nodularity. Cross-sectional imaging did not demonstrate any biliary or pancreatic ductal dilation, suspicious lymphadenopathy, or distant metastatic lesions and was otherwise unremarkable.

EGD/EUS demonstrated an 8-cm cystic lesion with septations but without nodules spanning from the head of the pancreas to the body of the pancreas. The pancreas parenchyma appeared heterogenous without masses or changes associated with chronic pancreatitis, the pancreatic duct was non-dilated, and the biliary system presented with no abnormalities.

An FNA was performed and cytologic findings revealed no malignant cells. Analysis of the fluid showed a carcinoembryonic antigen (CEA) level of $93.9 \mathrm{ng} / \mathrm{mL}$ and an amylase level of $222.5 \mathrm{IU} / \mathrm{L}$, both low, suggesting no malignancy.

The CT-CAP findings, along with EUS and the fluid anal- 


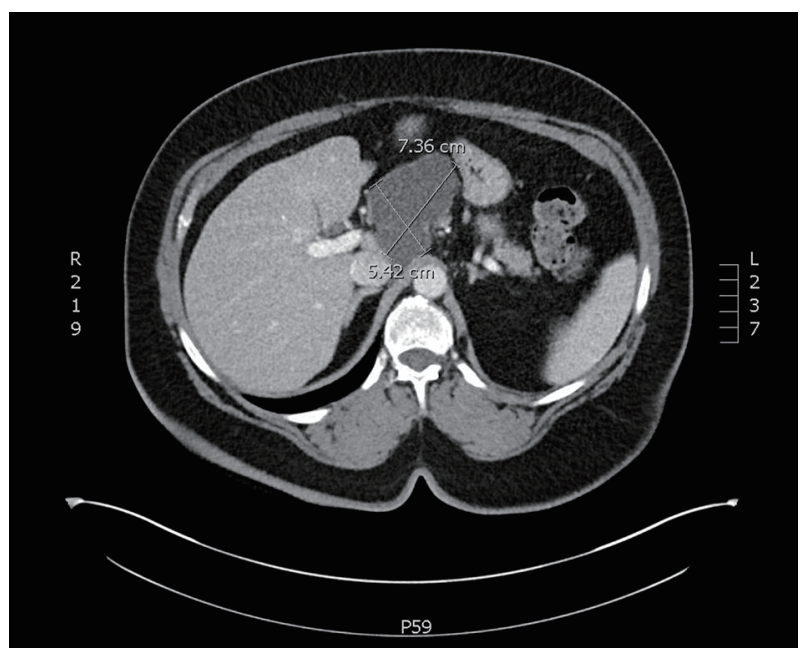

Figure 1. An axial slice from a CT scan with contrast. A cystic mass with few thin septae suggesting multi-loculation associated with the superior and anterior pancreatic body. CT: computed tomography.

ysis are consistent with pancreatic lymphangioma as they rule out other cystic lesions of the pancreas. Since the patient is currently asymptomatic, imaging studies will be used to monitor the lymphangioma.

\section{Outcome and follow-up}

A magnetic resonance imaging (MRI) scan 3 months later showed an interval decrease in size to $6.1 \times 4.3 \times 5.3 \mathrm{~cm}$. The cyst was a lobulated, macrocystic mass extending superiorly from the anterior pancreas body and draping over the portal vein and common hepatic artery while exerting a mass ef-

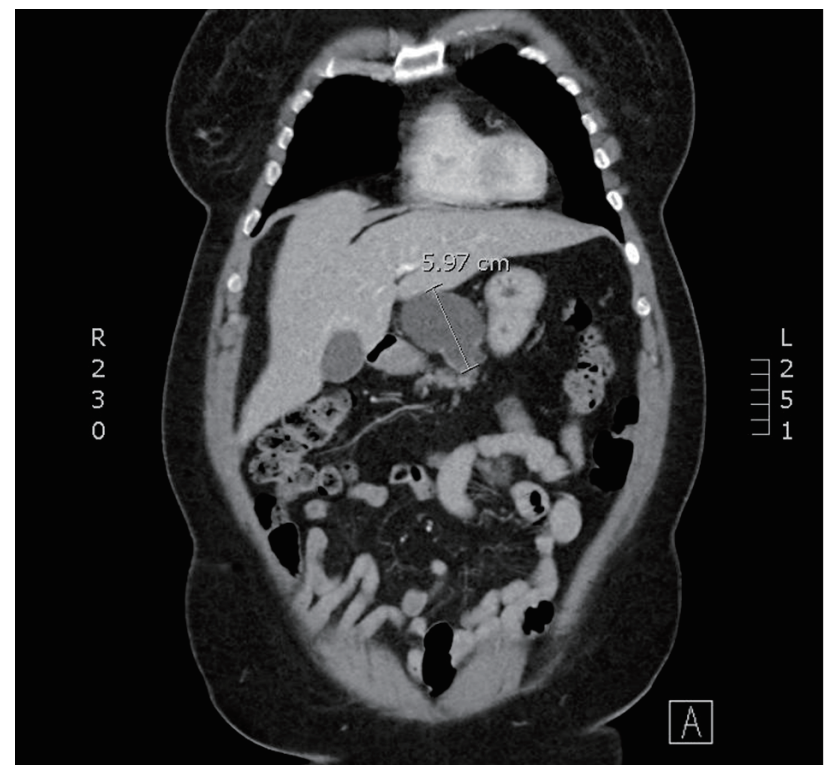

Figure 2. A coronal slice from a CT scan with contrast. A cystic mass with few thin septae suggesting multi-loculation associated with the superior and anterior pancreatic body. CT: computed tomography.

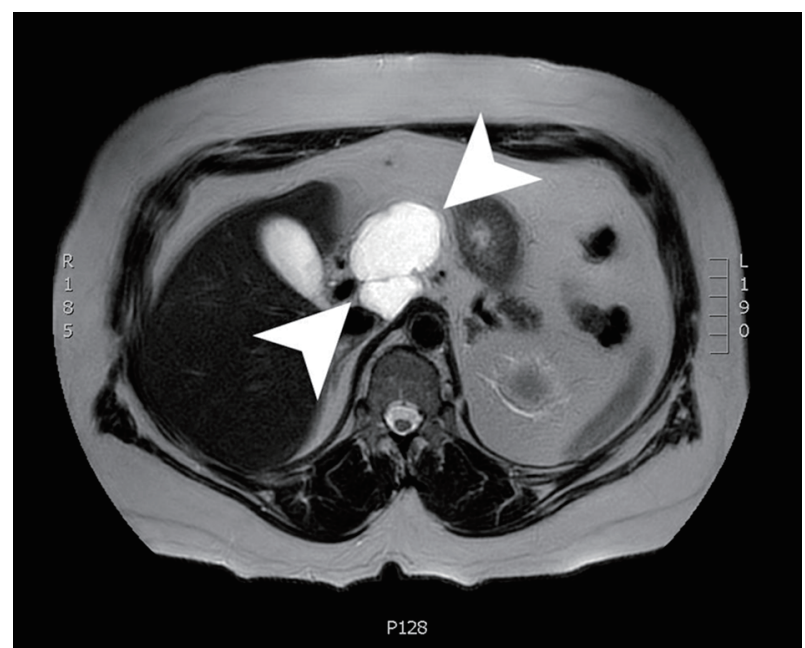

Figure 3. An axial slice from a T2-weighted MRI with gadolinium contrast showing a $6.1 \times 4.3 \times 5.3 \mathrm{~cm}$ lobulated, cystic mass with a few internal septations. MRI: magnetic resonance imaging.

fect on the posterior wall of the gastric antrum. The cyst wall demonstrated irregular enhancement with regions of restricted diffusion (Fig. 3). The patient was advised to follow up in 3 months with a CT scan to continue to monitor the size of the lesion. Due to the imaging and EUS characteristics, this lesion is felt to be a lymphangioma and will be followed.

\section{Discussion}

Lymphangiomas are benign, slow growing, rare tumors of the lymphatic system. Lymphangioma cases are mostly reported in children and are associated with a congenital malformation, such as trisomies 13, 18 and 21, Noonan syndrome, Turner syndrome and Down syndrome [7]. During embryogenesis, the disruption of lymphatic vessels results in lymph obstruction that is surrounded by a thin wall septae of endothelial cells [8]. This process gives rise to multiloculated lymphangiomas. Acquired lymphangioma can be a result of fibrosis, inflammation and lymphatic vessel degeneration [1].

Lymphangiomas account for about $5 \%$ of tumors in children and are commonly found in the neck region $(75 \%)$, followed by the axilla $(15 \%)[3,9,10]$. Presentation in adulthood is extremely rare, particularly in the pancreas, which accounts for less than $1 \%$ of all lymphangiomas [2]. The first reported case of pancreatic lymphangioma was described by Koch in 1913 [11]. Since then, to our knowledge, less than 90 cases have been reported in the English medical literature [12]. Pancreatic lymphangiomas are typically asymptomatic and discovered incidentally [5]. However, some patients may present with abdominal distention, diffuse abdominal pain and an associated abdominal mass [13-15]. Often, pancreatic lymphangiomas are diagnosed when they become symptomatic due to mass effect [8].

Lymphangiomas can be classified into capillary, cystic, or cavernous based on histological findings $[1,10,16]$ and these classifications were first described by Wegner in 1877 [17]. According to the 2018 International Society for the Study of 
Vascular Anomalies, cystic lymphangioma is also referred to as macrocystic lymphatic malformation $(>1 \mathrm{~cm}$ mean diameter), and cavernous lymphangioma is also referred to as microcystic lymphatic malformation $(<1 \mathrm{~cm}$ mean diameter). Therefore, lymphangiomas can be classified into microcystic, macrocytic and mixed lymphangiomas [18].

Capillary lymphangiomas are small, not-well defined vessels consisting of a high density of cellular stroma and are composed of small lymphatic vessels [10]. Microcystic lymphangiomas are associated with areas with dense connective tissue resulting in decreased muscle expansion, such as the lip and tongue. Macrocystic lymphangiomas arise in areas with loose connective tissue where expansion of endothelial-lined channels can occur, such as the neck, axilla and abdominal regions [19]. Macrocystic retroperitoneal lymphangiomas are mostly multilocular [10].

A diagnosis of lymphangioma is generally made based on clinical findings and imaging studies. Diagnostic imaging techniques include CT, MRI and EUS combined with FNA. Typical presentations are multilocular lesion with homogenous serous composition which appears anechoic on ultrasound. These lesions show a low signal intensity on T1-weighted images and very high signal intensity on heavily T2-weighted images. Furthermore, CT demonstrates fluid attenuation [20]. The imaging findings in this patient were consistent with a lymphangioma of the pancreas.

Historically, diagnosing lymphangiomas posed an issue due to the difficulty in differentiating pancreatic cystic lesions on imaging. The differential includes pseudocysts, serous cysts, intraductal mucinous neoplasms (IPMN) and mucinous cystic neoplasm (MCN) [21]. EUS-FNA technique has been an excellent tool to evaluate pancreatic cystic lesions and obtain a sample for analysis. Cystic fluid analysis for cytology, amylase and CEA can help differentiate benign from malignant cystic lesions. Typically, cystic fluid amylase is elevated in pseudocysts and IPMNs with CEA levels being low and elevated, respectively. On the other hand, serous cysts and MCNs have low cystic fluid amylase and low and elevated CEA, respectively [21, 22]. Lymphangiomas tend to have low levels of CEA, elevated levels of amylase and elevated levels of triglycerides [23].

The treatment can vary with the classification of lymphangioma. Spontaneous regression can occur in macrocystic or mixed lymphangiomas but not in the microcystic variant. Spontaneous regression is also more likely attributed to older patients [24]. Macrocystic and mixed lymphangiomas are pathologically similar and vary from microcystic lymphangioma in the balance of lymph fluid in-flow and out-flow [24]. Therefore, early treatment intervention in microcytic lymphangioma is a plausible approach due to the lack of spontaneous regression. If the patient is asymptomatic and the lesion is stable, a conservative approach is typically taken by frequent surveillance imaging. For definitive treatment of lymphangiomas, surgical resection is implicated. However, these benign lesions that do not need resection can be followed.

\section{Conclusion}

Pancreatic lymphangioma is a rare clinical finding in adults that often presents as an incidental diagnosis on imaging. It is an important differential to consider in any patient presenting with an atypical cyst of the pancreas. Imaging studies alone are often not conclusive and definitive diagnosis requires FNA analysis. Lymphangiomas are a benign lesion, therefore, if the patient is asymptomatic and the lesion is stable, complete surgical resection is not required and imaging surveillance is the preferred method of action. This case demonstrates the classic imaging findings of a lymphangioma in an unusual organ.

\section{Acknowledgments}

None to declare.

\section{Financial Disclosure}

None to declare.

\section{Conflict of Interest}

None to declare.

\section{Informed Consent}

Subject consent has been waived under the IRB approved study protocol and the identity of the subject has been protected.

\section{Author Contributions}

Sujata Ojha: acquisition of data, drafting of manuscript, critical revision; Muhammad Darwish: acquisition of data, drafting of manuscript, critical revision; Patrick McLaren: drafting of manuscript, critical revision; Annie Benzie: drafting of manuscript, critical revision; Edward Cho: drafting of manuscript, critical revision; Houssam Osman: drafting of manuscript, critical revision; Dhiresh Rohan Jeyarajah: study conception and design, drafting of manuscript, critical revision.

\section{Data Availability}

The authors declare that data supporting the findings of this study are available within the article.

\section{References}

1. Nuzzo G, Lemmo G, Marrocco-Trischitta MM, Boldrini G, Giovannini I. Retroperitoneal cystic lymphangioma. J Surg Oncol. 1996;61(3):234-237.

2. Colovic RB, Grubor NM, Micev MT, Atkinson HD, Rankovic VI, Jagodic MM. Cystic lymphangioma of the pancreas. World J Gastroenterol. 2008;14(44):6873- 
6875.

3. Park T, Lee HS, Jung EJ, Kim JY, Jeong CY, Ju YT, Lee YJ, et al. Concomitant breast and axillary lymphangioma in an adult: A case report and a review of the literature. Medicine (Baltimore). 2018;97(45):e12946.

4. Bihari C, Rastogi A, Rajesh S, Arora A, Arora A, Kumar N. Cystic lymphangioma of pancreas. Indian J Surg Oncol. 2016;7(1):106-109.

5. Carvalho D, Costa M, Russo P, Simas L, Baptista T, Ramos G. Cystic pancreatic lymphangioma - diagnostic role of endoscopic ultrasound. GE Port J Gastroenterol. 2016;23(5):254-258.

6. Igarashi A, Maruo Y, Ito T, Ohsawa K, Serizawa A, Yabe M, Seki K, et al. Huge cystic lymphangioma of the pancreas: report of a case. Surg Today. 2001;31(8):743-746.

7. Bansal AG, Oudsema R, Masseaux JA, Rosenberg HK. US of pediatric superficial masses of the head and neck. Radiographics. 2018;38(4):1239-1263.

8. Hussain I, Ang TL. Cystic pancreatic lymphangioma diagnosed with endoscopic ultrasound-guided fine needle aspiration. Endosc Ultrasound. 2017;6(2):136-139.

9. Bhavsar T, Saeed-Vafa D, Harbison S, Inniss S. Retroperitoneal cystic lymphangioma in an adult: A case report and review of the literature. World J Gastrointest Pathophysiol. 2010;1(5):171-176.

10. Rana A, Katzman PJ, Pegoli W, Qualia C. An unusual cause of abdominal pain: duodenal cystic lymphangioma. Gastroenterol Hepatol (N Y). 2013;9(3):192-195.

11. Koch K. Beitrage zur pathologie der Bauchspeicheldruse. Virchows Arch. 1913;214:180-206.

12. Fujii M, Saito H, Yoshioka M, Shiode J. Rare case of pancreatic cystic lymphangioma. Intern Med. 2018;57(6): 813-817.

13. Mansour NM, Salyers WJ, Jr. Recurrence of a pancreatic cystic lymphangioma after diagnosis and complete drainage by endoscopic ultrasound with fine-needle aspiration. JOP. 2013;14(3):280-282.
14. Schneider G, Seidel R, Altmeyer K, Remberger K, Pistorius $\mathrm{G}$, Kramann B, Uder $\mathrm{M}$. Lymphangioma of the pancreas and the duodenal wall: MR imaging findings. Eur Radiol. 2001;11(11):2232-2235.

15. Erguney S, Teksoz S, Erdamar S, Ainechii S, Olgun DC. Extended pancreaticoduodenectomy for a huge cysticcavernous lymphangioma: a case report. JOP. 2012; 13(3):289-291.

16. Shimada H, Ambros IM, Dehner LP, Hata J, Joshi VV, Roald B, Stram DO, et al. The International Neuroblastoma Pathology Classification (the Shimada system). Cancer. 1999;86(2):364-372.

17. Wegner G. Lymphangiome. Arch Klin Chirg. 1877; 20:641-707.

18. Dasgupta R, Fishman SJ. ISSVA classification. Semin Pediatr Surg. 2014;23(4):158-161.

19. Joshi PS, Hongal B, Sanadi A. Cystic lymphangioma: A differential diagnosis. J Oral Maxillofac Pathol. 2015;19(3):393-395.

20. Raufaste Tistet M, Ernst O, Lanchou M, Vermersch M, Lebert P. Imaging features, complications and differential diagnoses of abdominal cystic lymphangiomas. Abdom Radiol (NY). 2020;45(11):3589-3607.

21. Scholten L, van Huijgevoort NCM, van Hooft JE, Besselink MG, Del Chiaro M. Pancreatic cystic neoplasms: different types, different management, new guidelines. Visc Med. 2018;34(3):173-177.

22. Ngamruengphong S, Lennon AM. Analysis of pancreatic cyst fluid. Surg Pathol Clin. 2016;9(4):677-684.

23. Abdelkader A, Hunt B, Hartley CP, Panarelli NC, Giorgadze T. Cystic lesions of the pancreas: differential diagnosis and cytologic-histologic correlation. Arch Pathol Lab Med. 2020;144(1):47-61.

24. Kato M, Watanabe S, Kato R, Kawashima H, Iida T, Watanabe A. Spontaneous regression of lymphangiomas in a single center over 34 years. Plast Reconstr Surg Glob Open. 2017;5(9):e1501. 